\title{
Graph-based Analysis of Textured Images for Hierarchical Segmentation
}

\author{
Raffaele Gaetano 1,3 \\ raffaele.gaetano@sophia.inria.fr
}

\section{Giuseppe Scarpa ${ }^{2}$ \\ giscarpa@unina.it}

Tamás Szirányi $^{3}$
sziranyi@sztaki.hu

\author{
${ }^{1}$ ARIANA Project,
} INRIA Sophia Antipolis - Méditerranée, Sophia Antipolis, FRANCE

2 Dip. Ing. Biomed., Elett. e Telecom. Università degli Studi "Federico II", Napoli, ITALY

${ }^{3}$ SZTAKI - DEVA Group

Magyar Tudományos Akadémia, Budapest, HUNGARY

\begin{abstract}
The Texture Fragmentation and Reconstruction (TFR) algorithm has been recently introduced [ $[$ ] to address the problem of image segmentation by textural properties, based on a suitable image description tool known as the Hierarchical Multiple Markov Chain (H-MMC) model. TFR provides a hierarchical set of nested segmentation maps by first identifying the elementary image patterns, and then merging them sequentially to identify complete textures at different scales of observation.

In this work, we propose a major modification to the TFR by resorting to a graph based description of the image content and a graph clustering technique for the enhancement and extraction of image patterns. A procedure based on mathematical morphology will be introduced that allows for the construction of a color-wise image representation by means of multiple graph structures, along with a simple clustering technique aimed at cutting the graphs and correspondingly segment groups of connected components with a similar spatial context.

The performance assessment, realized both on synthetic compositions of real-world textures and images from the remote sensing domain, confirm the effectiveness and potential of the proposed method.
\end{abstract}

\section{Introduction}

The analysis and extraction of textural information from image data is a relevant topic in the image analysis and processing domains, mainly due to its transversality w.r.t. the addressed application areas. Apart from being of interest in the segmentation of natural images from the real world $[\theta]$, texture analysis ofter comes of great use also in more specific application

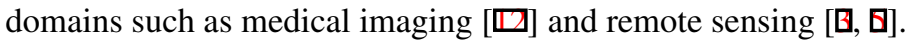

This work addresses the problem of hierarchical image segmentation driven by textural properties. The rationale that we follow here is based on the consideration that, especially in certain domains, an image can often be regarded as a complex composition of different 
textures, appearing at different scales and typically related to each other by hierarchical relationships. In particular, we here start from the framework introduced in [ $\mathrm{Q}]$, where a hierarchical texture modeling approach has been proposed, namely the Hierarchical Multiple Markov Chain model (H-MMC), along with the Texture Fragmentation and Reconstruction (TFR) algorithm for hierarchical image segmentation. Given the good potential expressed by these tools, we decided to carry on with their development by enriching the modeling approach with the use of a graph based image representation, to enhance the description of spatial interactions over the image and provide a robust support for the localization of texture patterns.

For the sake of brevity, considerations about the state of the art in hierarchical $/ \mathrm{multi}$-scale segmentation are left out here, referring the reader to [ $[$ ] for an extensive discussion on the subject. As for the use of graph structures in image analysis and segmentation, this is certainly not a novelty by itself: an early work in image processing [0] already proposed Region Adjacency Graphs as efficient tools to provide a "spatial view" of an image, and many works have been using them for segmentation as described in [ $\square$ ]. In [四] for example, starting from single pixels or elementary homogeneous regions (what is generally called superpixel) a complete segmentation is performed by associating similarity measures to graph edges and recursively merging nodes or extracting spanning trees. More recently, thanks to the breakthrough work of Shi and Malik [س]], a new approach to image segmentation based on global graph partitioning has been introduced, that opened to a new optimization framework based on the minimization of energy functions depending on graph measures. Extensions of this approach to the multiscale case have also been proposed, e.g. in [四], as well as many fundamental advances in the theoretical framework that can be found up to recent times [ $\square]$.

In this work, a multiple graph based representation is proposed, built starting from an initial partition of the image, and aimed at defining spatial relationships among elementary connected components, in order to discover similarities in the spatial context and extract texture patterns by means of a multiple graph clustering.

\section{Texture Fragmentation and Reconstruction (TFR)}

First we provide a brief recall to the reference modeling strategy and the related optimization method, in order to highlight their potential and show current limitations, referring the reader to [Q] for a more detailed description and experimental results.

Since its first formulation, the Hierarchical Multiple Markov Chain family of models has been proposed as a simple and effective tool to represent the visual properties of an image at any scale of observation, by means of its "broad sense" textural information: the core of the modeling strategy is the joint definition of texture components (patterns) at different scales and spatial interactions among them. The Texture Fragmentation and Reconstruction (TFR) algorithm has been then proposed as an actual hierarchical segmentation technique based on fitting image content into a H-MMC model. The TFR follows the well known split-and-merge paradigm: in a first fragmentation phase, clusters of elementary regions that share the same spectral and contextual properties are detected. These elementary patterns are then merged two-by-two during the reconstruction phase, giving rise to a hierarchy of nested segmentation maps at different scales of observation.

A flowchart of the algorithm is depicted in Fig. 1. It consists of three steps: a colorbased classification (CBC), a spatial context based clustering (SBC) and a texture merging phase. The first two steps constitute the fragmentation phase, while texture reconstruction 


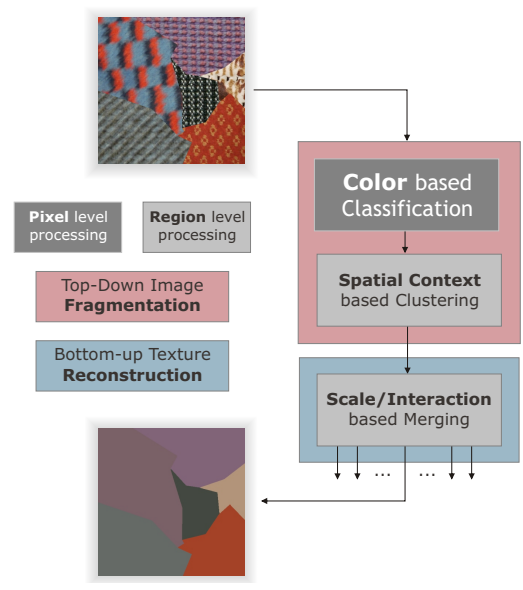

Figure 1: Flowchart of TFR.

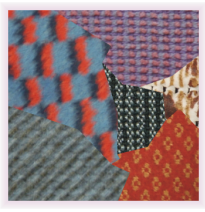

(a) data

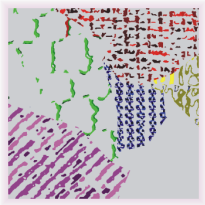

(d) partial SBC

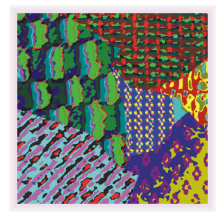

(b) $\mathrm{CBC}$

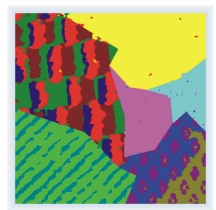

(e) 12 class map

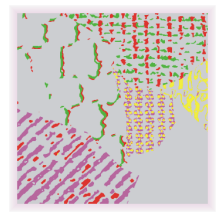

(c) partial $\mathrm{CBC}$

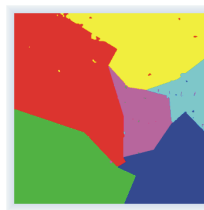

(f) 6 class map

Figure 2: A sample segmentation process.

is performed in the last step. The $\mathrm{CBC}$ is the only step of the procedure that works at pixel level, while subsequent steps move on the scale of connected components. The sequence of images in Fig. 2 ideally follows a sample segmentation process by showing its intermediate outputs, that will be discussed in the following.

Color Based Classification: this step carries out a color segmentation of the image; all pixels with the same color form a class, that in textured images is typically spread over the image giving origin to multiple color-homogeneous connected components. Color segmentation here is performed using the unsupervised TS-MRF algorithm described in [D], because of its favourable properties in dealing with noise and high-frequency irregularities. The number of color classes $K_{c}$, that represents a parameter value for TFR, is fixed a priori. In Fig. 2(b) the 24-class output of the CBC block for the sample texture mosaic of Fig. 2(a), taken from the Prague Texture Segmentation Benchmark [ $⿴ 囗 \mathbf{}]$, is shown. The choice of TSMRF is by no means critical: in principle any color segmentation algorithm capable to single out homogeneous regions and classify them by color could perform similarly, given that an excessive noise or a too high degree of fragmentation can reduce the quantity of information on spatial interactions.

Spatial Context Based Clustering: the aim of this block is readily appreciated by comparing Fig. 2(c), where a detailed view of 4 out of 24 color classes is shown, with Fig. 2(d). From the 4 color classes of the former, fragment groups are further split to obtain clusters of connected components that belong each to a single textured area.

To provide a similar decomposition, each fragment is characterized by means of a suitable description of its spatial context, and then a clustering in this feature space is operated, independently for each color class, to form groups of fragments of the same color that also share the same neighbourhood configuration. More precisely, given $\Omega$ the set of color classes available, $S_{\omega}$ a subset of pixels of the same color class $\omega \in \Omega$, and $\omega_{k}$ the label of its $k$-th connected component, the latter is associated with a set of probabilities:

$$
p_{j}\left(\omega^{\prime} \mid \omega_{k}\right)=\frac{\left|S_{\omega_{k} \stackrel{j}{\longrightarrow} \omega^{\prime}}\right|}{\left|S_{\omega_{k}}\right|}
$$

with $j \in\{N, N E, E, S E, S, S W, W, N W\}$, representing the likelihood of finding a pixel 
of color $\omega^{\prime}$ when moving from a location of the fragment $\omega_{k}$ in the direction $j$. The red fragments of the upper-left texture of Fig. 2(a), for example, will be characterized by a large probability of finding a blue pixel when leaving the region in the right direction and a black pixel when leaving to the left. The output of this block will be the finest scale segmentation map, with each segment consisting of a portion of the image homogeneous in terms of spectral/microtextural and contextual properties.

Scale/Interaction Based Texture Merging: this step has the goal of recovering all texture-coherent portions of the image, through multiple scales in a hierarchical fashion. This process consists of a sequence of binary region merging, selecting each time the image portion less likely to represent a complete and independent texture and merging it with its dominant neighbour, until the whole image is reconstructed. The core of this step is in the definition of the Texture Score $[\mathrm{\theta}]$, that basically measures the texture completeness of a current set of fragments $\xi$ by means of three different terms, respectively related to its area, compactness and nearest neighbour dominance:

$$
T S_{\xi}=p(\xi) \cdot \frac{1}{p(\bar{\xi} \mid \xi)} \cdot \frac{p(\bar{\xi} \mid \xi)}{\max _{\xi^{\prime} \neq \xi} p\left(\xi^{\prime} \mid \xi\right)}
$$

where $p(\bar{\xi} \mid \xi)$ is the probability of leaving the region $\xi$ in any direction and $p\left(\xi^{\prime} \mid \xi\right)$ the probability to reach the set $\xi^{\prime}$ in any direction. The described procedure generates the hierarchical stack of segmentation maps, the most meaningful ones appearing evidently in the top levels of the retrieved tree structure, as shown in Fig. 2(e) and 2(f) for the previous example where the 12- and 6-class maps are shown respectively.

\subsection{Addressing a weakness of TFR}

The TFR presents several interesting properties: it is able to recognize macro-texture at various scales of observation, it is unsupervised, and hence applicable in many different domains, and the region-based image modeling helps keeping the computational load reduced compared to other hierarchical segmenters. However, besides the good performances achieved by the TFR algorithm in different applications [ $[\mathbf{Q}, \mathbf{Q}]$, experimental evidence also pointed out several limitations and weak points that motivate further research. In this work we limit the scope of this analysis to the SBC block of Fig. 1.

The original SBC block is based on the independent featuring and clustering of connected components, without any specific spatial constraint: the features described in Eq. 1 are collected, and a reduction is performed using Principal Component Analysis. Clustering is realized using the $K$-means technique, by fixing a priori the number of desired clusters $K_{S}$ for each color class. The described choices generally lead to a quick and reliable output. However, in more complex cases, for example when color information is limited or in presence of significant scale differences, generally leading to largely unbalanced clusters, the $K$-means clustering is likely to perform unsatisfactorily, mainly due to the following reasons:

- statistical clustering needs a large number of samples to be reliable: in case of highly unbalanced "actual" clusters, $K$-means can easily give rise to a seriously erroneous clustering;

- the necessity of compacting data to cope with the curse of dimensionality reduces the accuracy of context description; 


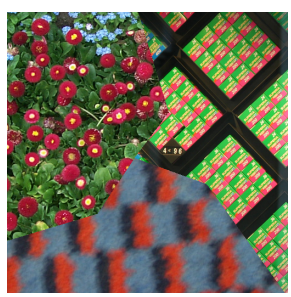

(a)

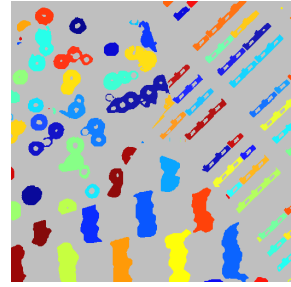

(b)

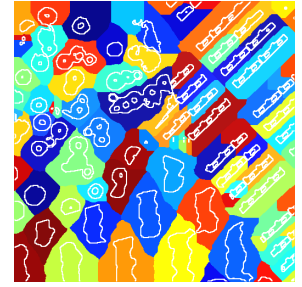

(c)

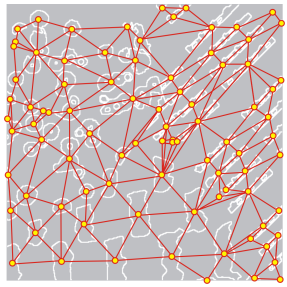

(d)

Figure 3: Toy example of the color-wise graph construction. In 3(a) a sample texture mosaic is shown, whose red subset is highlighted in 3(b) where each connected component (fragment) has a different label. In 3(c), the fragment map after label propagation is shown, and the relative RAG is depicted in $3(\mathrm{~d})$.

- in the absence of spatial constraints, no control is provided on cluster localization in space, thus renouncing to a consistent information, since fragments of the same texture evidently are "close" to each other with high probability.

In the following section, we propose a completely new implementation for this block, where the clustering approach is no longer divisive but agglomerative. This is here obtained by switching to graph structures, that keep track of the proximity among fragments and help measuring context differences locally instead of relying on global statistics.

\section{Proposed Method}

The main rationale for the method introduced here concerns the importance of spatial proximity in forming intra-textural patterns from a group of fragments. Including spatial location as a feature in the existing scheme is not reasonable, both because the varying shapes of fragments make any possible position feature inaccurate, and because the feature space dimension would be even higher compromising the reliability of clustering.

Our idea, then, is to associate to each map of color-uniform fragments a graph based representation that allows for the definition of neighbourhood relationships. This superimposed topology will provide additional information about the spatial localization of clusters, and hence drive cluster formation so as to avoid, for example, to merge clusters that are separated by unrelated textures.

To achieve this goal, two main issues have to be solved. First of all, the construction of the graph structure is not trivial, since the fragments are generally disconnected and we cannot unambiguously determine "who neighbours who" in this context (consider a map like the one of Fig. 3(b)). Furthermore, once this topology is defined, we need to choose suitable metrics to measure context differences and deploy a fast and effective graph clustering technique.

\subsection{Building the graph based image representation}

The graph based representation introduced here relies on the definition of multiple graphs, associated one-to-one with the color classes of the image. Hence, the starting point for the analysis of spatial contexts is again the color classification described in Sect. 2. 
Let us go directly into details by following the toy example of Fig. 3: in Fig. 3(a), a mosaic of real textures is presented, where the red color has been extracted and the corresponding connected components are shown in the map of Fig. 3(b), independently labeled.

The idea is to build a graph $G_{r e d}(V, E)$ where each node $v_{i}$ is associated to a fragment and a link exists between two nodes $v_{i}, v_{j}$ if the corresponding fragments are neighbours in some sense. Since the fragments are all disjoint by definition, a strategy is needed to define neighbouring relationships: here, we opt for the generation of suitable adjacencies among fragments, by resorting to a uniform-speed label propagation starting from the partial fragment map as the one in Fig. 3(b). This procedure, inherited from mathematical morphology [प]], is very simple and can be resumed as follows:

1. Starting from the fragment map $X$, initialize a queue with all labeled pixels (i.e. belonging to some fragment) that have at least a non-labeled neighbour;

2. pick a pixel $p$ (of label $x_{p}$ ) from the queue and check its neighbourhood $\mathscr{N}_{p}$ : for any non labeled pixel $q \in \mathscr{N}_{p}$, set $x_{q}=x_{p}$ and add $q$ to the queue;

3. repeat step 2 until the queue is empty.

The pixel-wise label propagation is here obtained using a 4-connected structuring element. The result of such procedure on the example of Fig. 3(b) is shown in Fig. 3(c), where the fragment contours are superimposed. A graph representation of the red color class is now easily built by computing the region adjacency graph [ $[$ ] of this map (see Fig. 3(d)).

\subsection{The Linked Spatial Based Clustering (L-SBC)}

The new SBC block here proposed relies on a modified definition of finest-scale pattern: beside being a set of spectrally homogeneous connected regions exhibiting similar spatial contexts (according to the probabilities of Eq. 1), we also require each of these patterns to be "connected", in the sense of the graph based representation introduced in Sect. 3.1. This basically means that the subgraph associated with a set of fragments of a certain color class that form an elementary pattern must be connected. To provide such decomposition, a simple agglomerative clustering technique has been developed that is based on local context similarity measures, namely the Linked Spatial Based Clustering (L-SBC). This procedure runs in parallel on all color classes and comprises the following steps:

1. for each color class $\omega$ compute the graph $G_{\omega}$;

2. for each graph $G_{\omega}$, annotate its links $\left\{E_{i j}^{\left(G_{\omega}\right)}\right\}_{i \neq j}$ using of a context similarity measure $C S_{i j}$ between the fragments $\omega_{i}$ and $\omega_{j}$;

3. given the total number of links from all the graphs, determine the threshold $C S_{t h}$ above which only a fixed percentage of the links is retained, say $\left.\psi_{L} \in\right] 0,1[$;

4. for each graph, remove all the links $E_{i j}$ for which $C S_{i j}<C S_{t h}$, determine the connected subgraphs (including single nodes) and label accordingly the corresponding fragments.

The metric $C S_{i j}$, derived from the probabilities of Eq. 1, is defined as follows:

$$
C S_{i j}=\frac{1}{\left[\frac{1}{8(K c-1)} \sum_{r, s}\left(A_{r s}^{i}-A_{r s}^{j}\right)^{2}\right] \cdot\left[\frac{1}{K c-1} \sum_{s}\left(\frac{1}{8} \sum_{r} A_{r s}^{i}-\frac{1}{8} \sum_{r} A_{r s}^{j}\right)^{2}\right]},
$$




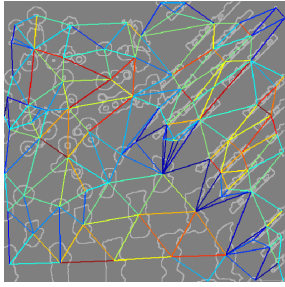

(a)

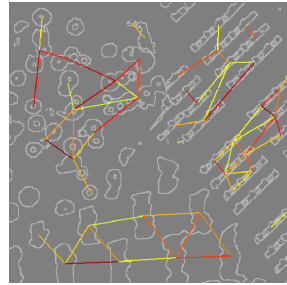

(b)

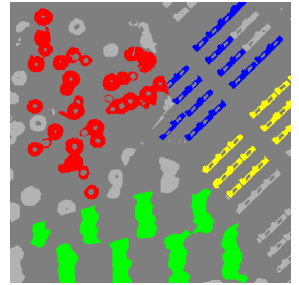

(c)

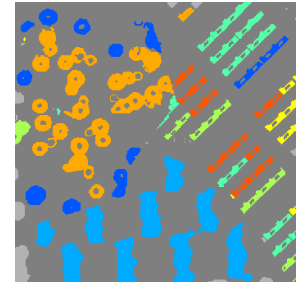

(d)

Figure 4: L-SBC example on the red subset of the image in Fig. 3(a): the graph with strongest links in hot colors (4(a)), the effect of link thresholding with $\psi_{L}=0.1$ (4(b)), the four most significant clusters retrieved (4(c)) and the most significant clusters obtained with the old $k$-means based method (4(d)).

where $A_{r s}^{k}$ is a $8 \times\left(K_{c}-1\right)$ matrix whose element $(r, s)$ is corresponding to $p_{r}\left(s \mid \omega_{k}\right)$ in Eq. 1, with $s \in\left\{1, \ldots, K_{c}-1\right\}$ being an index of the set $\left\{\gamma \in \Omega: \omega_{k} \notin \gamma\right\}$, that is, selftransition probabilities are excluded. The latter choice is due to the fact that a self-transition probability, that is, the probability of remaining inside the fragment when moving one pixel from a fragment's location, are usually of a higher order of magnitude w.r.t. the others, and if included would limit the weight of context description in the two Mean Square Errors at the denominator of Eq. 3. The second MSE is included to enhance context similarities between fragments that interact with the same colors but in a different configuration (think of isotropic and/or non-structured textures). The choice of the percentage $\psi_{L}$ is directly related to the number and scale of the different patterns present in the image, and is made robust by the fact that an image pattern can be rebuilt even if not all of its links are selected. In Fig. 4 the entire procedure is shown step-by-step for the sample mosaic of Fig. 3(a). Notice that the old SBC produces some under-segmentation errors (mis-clustered fragments) also for this simple case with highly biased contexts. On the other hand, L-SBC can cause some oversegmentation, like for the upper right cluster of Fig. 4(c), but this is likely to be compensated in the merging phase by the presence of full patterns that act as collectors [ $\mathrm{\theta}]$.

\section{Experimental Results}

A first validation of the new version of TFR with L-SBC has been performed on the Prague Segmentation Benchmark [ $[$ ]. For each of the 20 mosaics of the dataset, segmentation has been performed and the best map has been manually chosen from the hierarchical stack ${ }^{1}$. The two algorithms are identical but for the SBC block; relevant parameters are set to $K_{c}=24$ for both cases, $K_{S}=12$ for old SBC, $\psi_{L}=0.1$ for L-SBC, estimating a $10 \%$ of "useful" links over the whole graph representation. Some of the results are reported in Fig. 5 for the old and new version of the algorithm. For these segmentations, a numerical evaluation is also available that shows how the new algorithm significantly outperforms the old version ${ }^{2}$, with a Correct Segmentation (CS) index (refer to http://mosaic.utia.cas.cz/ for details on the indicators) that goes from $51.25 \%$ to $65.15 \%$ and the other values confirming this quality leap (GCE from 20.35 to 17.66 , LCE from 14.86 to 12.83 , Noise Errors (NE)

\footnotetext{
${ }^{1}$ The opportunity of this choice for the presentation of TFR results is discussed in [ $\left.\mathbf{\theta}\right]$.

${ }^{2}$ The reference method is indicated as TFR+ in [ $[\boldsymbol{\theta}]$ and uses an enhanced Texture Score based on KarhunenLoeve Divergence. At present, it provides the best results on the Prague Benchmark.
} 

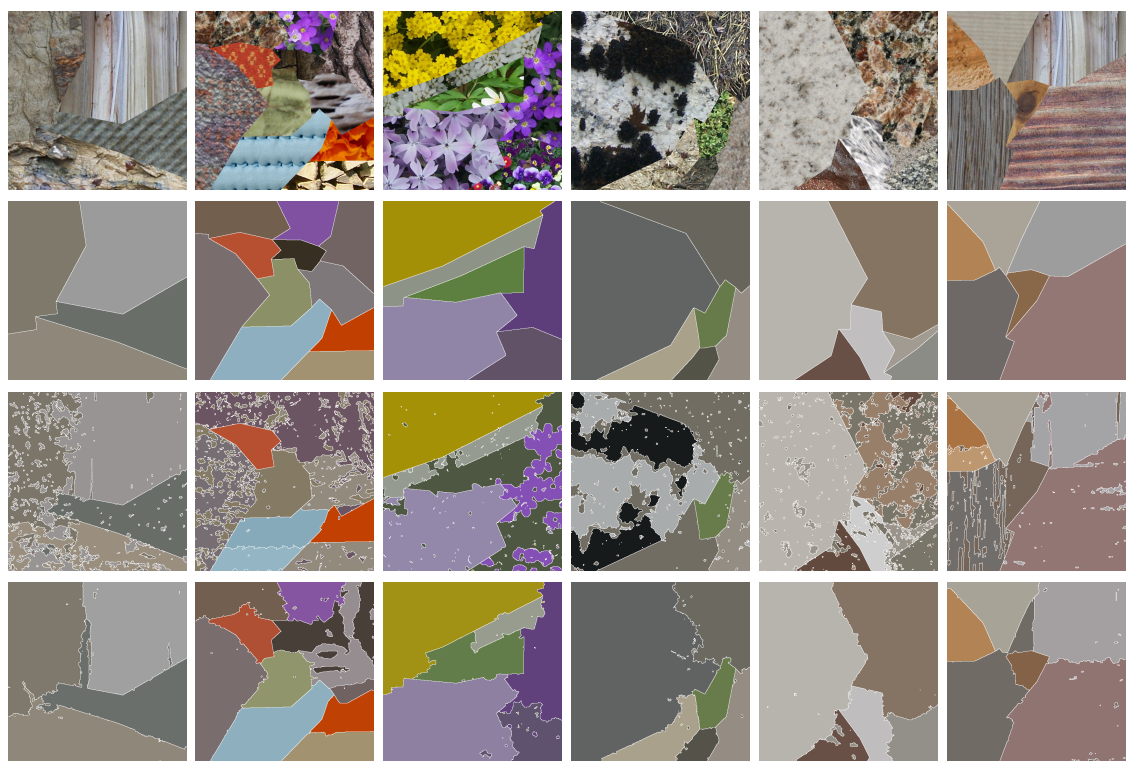

Figure 5: Results of TFR algorithm on several texture mosaics from the Prague Benchmark. First row: source texture mosaics. Second row: ground truths. Third and fourth rows: best segmentation results using TFR+ and new results obtained using TFR w/L-SBC.
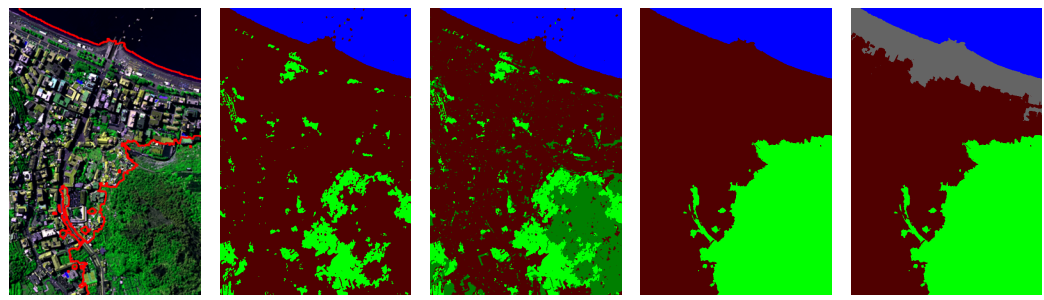

Figure 6: An aerial image of Minori (NA, Italy, (C)AvioRiprese S.R.L.) and the corresponding 3- and 4-class segmentation respectively with classical TFR and TFR w/L-SBC.

from 31.38 to 25.59). These improvements are mainly due to the facts that the L-SBC block succeeded in keeping the elementary texture patterns well localized in the image while the classical SBC tends to produce an excessive spread or noisy clusters, generating many noise errors and having a non negligible impact on the subsequent merging phase. This is testified by the higher degree of irregularity of classical TFR maps and the general tendency to undersegmentation. A slight increase in the total running time has been experienced, but the overall computational load remains limited (a $512 \times 512$ image is processed in 23 to 25 seconds with this version against an average of 20 for the old one, on a machine equipped with a $1.66 \mathrm{GHz}$ processor).

As a further assessment, we also show here some experiments on very high resolution images from the remote sensing domain, where TFR already proved to be an effective tool for multiscale image classification [ $\mathrm{G}$. Results are shown in Fig. 6 and Fig. 7, where contours of the segmentation maps obtained with the new technique are superimposed to the source image. Such maps are again manually picked from the hierarchical set. The first experiment (Fig. 6) concerns an aerial image of an area around Naples, Italy (size $500 \times 750$, 

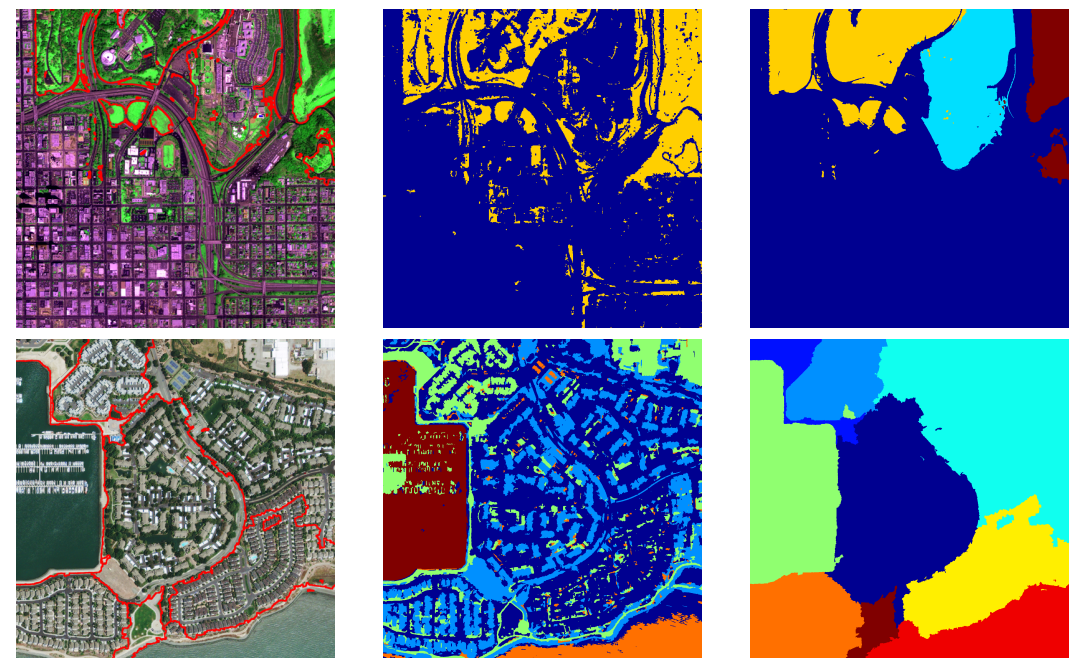

Figure 7: First row: an image of San Diego (CA, USA, (C)IKONOS) from the Ikonos satellite, and the urban-peripheral classification provided respectively by the old and new algorithm. Second row: a GeoEye-1 image of San Francisco (CA, USA, (C)GEOEYE), with the respective segmentations (old TFR and TFR w/L-SBC) of several intra-urban environments.

4 bands with a spatial resolution of around $80 \mathrm{~cm}$ ) exibiting 3 main classes (sea, urban area and hillside). Again, the number of initial color classes is fixed to $K_{c}=8$ for both algorithms, with $K_{s}=5$ for SBC and $\psi_{L}=0.1$ for L-SBC (this set has been kept also for the next experiments). Classical TFR (second and third image) fails to correctly detect inland classes, mainly because of the lack of color information that limits the reliability of contextual features, while the new version correctly isolates the hillside area and, one level below on the hierarchy, also provides the segmentation of the seashore, that clearly exhibits different textural properties. In Fig. 7 (first row), urban area segmentation of an image provided by the Ikonos satellite is presented $(2004 \times 2004$ pixels, 4 pansharpened bands with $1 \mathrm{~m}$ spatial resolution), with the classical TFR providing a good result for binary classification, basically including all urban and peripheral parts in one class and outer vegetation in the other, while the new technique identifies, along with the urban class including main roads, a separated class comprising the industrial area in the north, and two different vegetation areas. In the end, a third experiment is presented in the second row of Fig. 7, on a challenging GeoEye1 image exhibiting several different intra-urban environments $(1600 \times 1600$ pixels at $45 \mathrm{~cm}$ resolution). Here, the old TFR fails, getting stuck on fine details mainly because the majority of the detected elementary texture patterns are spread all over the image. Very interesting in this case is the segmentation provided by the new algorithm, where the main environments have been neatly detected according to their visual aspect, with the only possible oversplit of the central and top-right areas, whose fragments are characterized by a different orientation.

\section{Conclusions}

In this work, we have presented some advances in the Texture Fragmentation and Reconstruction algorithm, a recently proposed hierarchical image segmentation algorithm. A 
technique for the detection of elementary texture patterns with homogeneous spectral and contextual properties has been developed that relies on the use of graph structures. The algorithm starts from a suitable partition of the image in color/microtextural classes, and works independently for each class. Given a partial class map that comprises a series of color-homogeneous connected components, thanks to the morphological construction of region adjacencies, a graph is built where each node represents a fragment and a link exists if two fragments are "neighbours". These links are then annotated by measuring the context similarity between the fragments associated with the link vertices. Graph cuts, obtained by removing links below an automatically determined threshold, carry out the texture patterns. Integration of this technique into the TFR algorithm has proved successful, providing promising results both on the Prague Segmentation Benchmark and on real-world images from the remote sensing domain. Further research is under way to optimize the graph clustering. The definition of a new texture score metric, which take into account the color-wise graph structures introduced here, will also be object of future works.

\section{Acknowledgements}

This work has been carried out during the tenure of a post-doctoral fellowship (Gaetano's post-doc) promoted by the ERCIM consortium, which the authors would like to thank.

\section{References}

[1] T. Cour, F. Bénézit, and J. Shi. Spectral segmentation with multiscale graph decomposition. Proc. of IEEE Conference on Computer Vision and Pattern Recognition CVPR 2005, 2:1124-1131, June 2005.

[2] C. D'Elia, G. Poggi, and G. Scarpa. A tree-structured Markov random field model for Bayesian image segmentation. IEEE Trans. on Im. Proc., 12(10):1259-1273, October 2003.

[3] R. Gaetano, G. Scarpa, and G. Poggi. Hierarchical texture based segmentation of multiresolution remote sensing images. IEEE Trans. on Geo. and Rem. Sens., 47(7):21292141, August 2009.

[4] M. Haindl and S. Mikeš. Texture segmentation benchmark. In Proceedings of the 19th International Conference on Pattern Recognition, ICPR 2008, pages 1-4, Los Alamitos, December 2008. IEEE Computer Society. ISBN 978-1-4244-2174-9. doi: http://dx.doi.org/10.1109/ICPR.2008.4761118.

[5] A. S. Laliberte and A. Rango. Texture and scale in object-based analysis of subdecimeter resolution unmanned aerial vehicle (uav) imagery. IEEE Transactions on Geoscience and Remote Sensing, 47(3):761-770, 2009.

[6] T. Pavlidis. Structural Pattern Recognition. Springer, New York, 1980.

[7] Anil Raj and Chris H. Wiggins. An information-theoretic derivation of min-cut-based clustering. IEEE Transactions on Pattern Analysis and Machine Intelligence, 32(6): 988-995, 2010. 
[8] G. Scarpa, R. Gaetano, and G. Poggi. Texture image segmentation by hierarchical modeling. In Proc. European Signal Processing Conference, EUSIPCO 2008, Lausanne $(\mathrm{CH})$, August 2008.

[9] G. Scarpa, R. Gaetano, M. Haindl, and J. Zerubia. Hierarchical multiple markov chain model for unsupervised texture segmentation. IEEE Trans. on Im. Proc., 18(8):18301843, August 2009.

[10] J. Shi and J. Malik. Normalized cuts and image segmentation. IEEE Transactions on Pattern Analysis and Machine Intelligence, 22(8):888-905, Aug. 2000.

[11] P. Soille. Morphological Image Analysis, Principle and Applications, 2nd ed. SpringerVerlag, 2002.

[12] L. Srensen, S. B. Shaker, and M. de Bruijne. Quantitative analysis of pulmonary emphysema using local binary patterns. IEEE Transactions on Medical Imaging, 29(2): 559-569, 2010.

[13] A. Tremeau and P. Colantoni. Regions adjacency graph applied to color image segmentation. IEEE Transactions on Image Processing, 9(4):735-744, 2000.

[14] T. Vlachos and A. G. Constantinides. Graph-theoretical approach to colour picture segmentation and contour classification. IEE Proceedings I Communications, Speech and Vision, 140(1):36-45, 1993. 